\title{
La etnografía del comportamiento ecológico y su relación con la cultura ambiental en pobladores del distrito Huancas, provincia Chachapoyas, región Amazonas
}

\author{
Alex Alonso Pinzón Chunga \\ Alex.pinzon@untrm.edu.pe \\ Roberto Esteban Sánchez Colina \\ rsanchez@unsm.edu.pe \\ Fidel Ernesto Crisanto Gómez \\ Fidel.crisanto@untrm.edu.pe
}

\section{RESUMEN}

Se realizó una investigación con estudiantes de la Escuela Profesional de Antropología de la Facultad de Ciencias Sociales de la Universidad Nacional de Toribio Rodríguez de Mendoza, en la provincia y departamento de Amazonas, con el objetivo de determinar el grado de relación entre cultura y naturaleza de los pobladores del distrito de Huancas en provincia de Chachapoyas 2016. Los datos se obtuvieron mediante Encuestas y entrevista estructurada a una muestra seleccionada de pobladores. Para el procesamiento de los datos se utilizó la estadística descriptiva, con medidas resúmenes para variables cualitativas, valores absolutos y porcentajes. Los resultados se representaron en tablas, gráficos estadísticos, que permitieron una mejor interpretación y la elaboración de conclusiones y recomendaciones. Se comprobó la existencia de deficiencias del comportamiento ecológico en los pobladores, esto a su vez están relacionados en su cultura ambiental, lo que podrá ser de gran valor para el desarrollo de acciones en educación ambiental.

Palabras clave: etnografía; cultura; naturaleza; comportamiento ecológico; dimensiones. 


\title{
The ethnography of ecological behavior and its relationship with environmental culture in residents of the Huancas district, Chachapoyas province, Amazonas region
}

\begin{abstract}
ABSTRAC
An investigation was carried out with students from the Professional School of Anthropology of the Faculty of Social Sciences of the National University of Toribio Rodríguez de Mendoza, in the province and department of Amazonas, with the aim of determining the degree of relationship between culture and nature of residents of the district of Huancas in the province of Chachapoyas 2016. The data were obtained through surveys and structured interviews with a selected sample of residents. Descriptive statistics were used for data processing, with summary measures for qualitative variables, absolute values and percentages. The results were represented in tables and statistical graphs, which allowed a better interpretation and the elaboration of conclusions and recommendations. The existence of deficiencies in ecological behavior in the inhabitants was verified, this in turn is related to their environmental culture, which may be of great value for the development of actions in environmental education.
\end{abstract}

Keywords: ethnography; culture; nature; ecological behavior; dimensions.

Artículo recibido: 02 Setiembre. 2021 Aceptado para publicación: 30 Setiembre. 2021 Correspondencia: Fidel.crisanto@untrm.edu.pe Conflictos de Interés: Ninguna que declarar 


\section{INTRODUCCIÓN}

La actual preocupación mundial sobre el estado del medio ambiente ha conllevado un aumento espectacular del interés académico por los asuntos medioambientales, lo cual ha afectado tanto las ciencias sociales como las ciencias físicas. En el ámbito de la antropología, así como en algunas otras disciplinas, el interés por los modos en que la gente se relaciona con el medio ambiente no es nuevo: los antropólogos especializados en este campo se han referido a menudo a una antropología de orientación ecológica que se ha dedicado a estudiar dicha relación durante los últimos cien años aproximadamente. "La severa crisis ambiental que enfrentamos se debe a factores concurrentes como el vertiginoso incremento de la población humana, aunado al acelerado crecimiento de todas las actividades económicas que han producido impactos negativos en el ambiente" (Westreicher, 2016, p. 25). Por su parte la cultura ambiental en los pobladores del distrito de Huancas es un problema que se debate mucho, pues la mayoría consideran que este se debe a la falta de inteligencia ecológica.

En el Perú la importancia del comportamiento ecológico es determinante, pues representan el eje central que contribuye alcanzar el buen vivir, la salud pública el desarrollo sostenible de la vida. La falta de inteligencia ecológica en los pobladores del distrito de Huancas es un factor para desarrollar un acercamiento con la educación ambiental. La educación ambiental ha permitido en estos últimos treinta años crear un comportamiento ecológico que genere el cuidado del medio ambiente.

El tipo e intensidad de la cultura ambiental que desarrollan los habitantes del distrito de Huancas está condicionado, entre otros factores, por el medio social en el que viven, y puede llevarles a adoptar patrones de comportamiento que fomentan el cuidado del ambiente. Ecología práctica el nuevo sueño para sumar esfuerzos, generar un cambio de mentalidad y un comportamiento más amigable con el ambiente. (Miro Quesada, 2015, p. $03)$.

Este estudio investiga la relación del comportamiento ecológico con las creencias ambientales. Se ha seleccionado una determinada muestra de pobladores del distrito, dirigido a la población en general y centros educativos; quienes respondieron a dos cuestionarios. El primero mide el comportamiento ecológico (limpieza urbana, ahorro del agua y de energía, activismo y reciclaje) y el segundo mide creencias ambientales (ecos céntricos y antropocéntricos). 
Además, se obtiene un factor general llamado de Comportamiento Ecológico General, que es la media de los cuatro factores específicos. Las creencias ecos céntricos y antropocéntricos son predictores de los factores limpieza urbana y ahorro del agua y de energía, y la edad predice el reciclaje. Por tanto, los resultados indican que las creencias ambientales son antecedentes del comportamiento ecológico entre los pobladores del distrito de Huancas.

La región Amazonas es un típico departamento rural del Perú, enclavado entre la costa y la selva norte. La mayoría de su población aún vive en el campo. Cuando estamos en el distrito de Huancas, la ruralidad de la región Amazonas se expresa con todo su esplendor. Una nueva propuesta de desarrollo, debe ser pensada desde el culturalismo, no es utópica ni descabellada, pero ahora en manos de los propios amazonenses, debe tomar en cuenta este aspecto y revalorarlo, si queremos que Amazonas deje de ser visto sólo como un departamento de "paso" entre la costa y la selva y sea un lugar donde quedarse y echar raíces. Un lugar donde vivir el futuro de nuestro país. ¿Cuál es el grado de relación entre el comportamiento ecológico y la cultura ambiental en los pobladores del distrito de Huancas, provincia de Chachapoyas, Amazonas 2016?

\section{ESTRATEGIAS METODOLÓGICAS O MATERIALES Y MÉTODOS}

El enfoque es cuantitativo, el tipo de investigación es no experimental y el método de la investigación será de acuerdo a lo explicado por los autores Hernández, Fernández Bautista (2014), esta metodología de investigación comprende la realización de una sola evaluación, en su estado natural es decir sin manipular la conceptualización teórica de las variables; tomando en cuenta las dimensiones e indicadores propuestas por cada autor.

De tal forma, el diseño de la investigación será descriptiva. Examina la relación o asociación de variables en la misma unidad de investigación (Hernández, Fernández y otros, 2014); asimismo, para la presente investigación se empleó como técnica la encuesta y para la recolección de los datos se utilizó como instrumentos, dos cuestionarios referidos a las variables de la investigación 


\section{RESULTADOS Y DISCUSIÓN}

Motivación en el cuidado ambiental de pobladores del distrito Huancas.

\section{Tabla 1}

Motivación en el cuidado ambiental en pobladores del distrito Huancas.

\begin{tabular}{|c|c|c|}
\hline Escalas de medición & Frecuencia & Porcentaje \\
\hline Muy alta & 14 & $13 \%$ \\
\hline Alta & 26 & $25 \%$ \\
\hline Regular & 33 & $32 \%$ \\
\hline Bajo & 30 & $29 \%$ \\
\hline Muy Bajo & 0 & 0.00 \\
\hline Total & $\mathbf{1 0 3}$ & $\mathbf{1 0 0 \%}$ \\
\hline
\end{tabular}

Fuente: Elaboración propia

Se observa que en los pobladores del distrito de Huancas - Chachapoyas, los pobladores no presentan una motivación adecuada ya que el 32\% de los 103 encuestados así lo confirman, esto debido a que consideran que las tareas ambientales no están claramente definidas ni lógicamente estructuradas por parte de las autoridades locales, además no están de acuerdo con los procedimientos que desarrollan para el cumplimiento con el cuidado ambiental. En una entrevista con un comunero del distrito de Huancas nos manifestaron lo siguiente: las siembras que se hacen todo el año, se hacen en terrenos húmedos, siendo la humedad un factor que beneficia el crecimiento de un producto agrícola. Otras personas afirman que sus siembras se realizan, mayormente en épocas de lluvias y principalmente en los meses como septiembre y octubre. En estos meses tienen productos agrícolas adecuados como el maíz, papas, trigo y habas, de estos dependerá la producción local, ya que se realizan para satisfacer necesidades básicas como alimentación y en menor medida, para sacar al mercado en beneficio económico. Los meses en los cuales realizan la siembra, forma parte esencial de la cultura de Huancas. Si se toma en consideración las costumbres de la localidad, los pobladores no toman en cuenta otros meses por reconocerlos como tiempos de sequía y muchas veces ese tipo de conocimiento no universitario choca con las disposiciones de las autoridades locales y muchas veces promueven que no nos pongamos de acuerdo. 


\section{Inteligencia ecológica de los pobladores del distrito de Huancas.}

\section{Tabla 2}

Inteligencia ecológica de los pobladores del distrito de Huancas.

\begin{tabular}{|c|c|c|}
\hline Escalas de medición & Frecuencia & Porcentaje \\
\hline Muy alta & 10 & $10 \%$ \\
\hline Alta & 18 & $17 \%$ \\
\hline Regular & 35 & $34 \%$ \\
\hline Bajo & 30 & $29 \%$ \\
\hline Muy Bajo & 10 & $10 \%$ \\
\hline Total & $\mathbf{1 0 3}$ & $\mathbf{1 0 0 \%}$ \\
\hline
\end{tabular}

Fuente: Elaboración propia

La inteligencia ecológica en los pobladores del distrito de Huancas- Chachapoyas es regular en un $34 \%$. Todo esto debido a que manifiestan que existe un choque cultural entre sus costumbres con el medio ambiente y las costumbres oficialistas de las autoridades ambientales que no se dejan entender o en su defecto no solucionan los problemas prácticos ambientales, eso limita el completo entendimiento de muchos temas ambientales impartidos durante décadas en el distrito de Huancas. El distrito de Huancas, no es ajeno a problemas de esta índole, las creencias ecológicas y educación ambiental que trasciende desde la educación en casa, y la educación en diversas instituciones forman unas concepciones de cada uno de los pobladores, muchas veces atentatorias con la naturaleza. En base a la Zonificación Ecológica Económica, la localidad de Huancas está ubicada en la zona de vida denominada "Bosque seco Montano Bajo Tropical". El cual se encuentra localizada entre 2.000 y $3.000 \mathrm{~m}$, con variaciones micro-climáticas de acuerdo a los pisos altitudinales de las cordilleras. Representa el $21 \%$ del área de estudio. La vegetación primaria de esta formación ha sido alterada completamente. En la actualidad se observan muy pocas asociaciones de árboles y muchas áreas de cultivos de subsistencia. En algunas zonas se localizan formaciones de eucaliptos, cipreses y pinos. La vegetación natural clímax ha desaparecido casi por completo a través de los años para ser remplazada por cultivos y dar a una vegetación secundaria constituida por gramíneas, arbustos y árboles dispersos. 
En el distrito Huancas de la provincia de Chachapoyas, se resumen a pocos datos o escasa bibliografía documental sobre etnografía cultural, este trabajo nos motiva investigar y dar conocimiento a la cultura contemporánea de los pobladores Huancas.

\section{Medida de adecuación muestral de Kaiser-Meyer-Olkin (KMO) y prueba de Bartlett}

Tabla 3

Medida de adecuación muestral de Kaiser-Meyer-Olkin (KMO) y prueba de Bartlett

\begin{tabular}{lcc}
\hline \multicolumn{2}{l|}{ Medida de adecuación muestral de Kaiser-Meyer-Olkin. } & 0.974 \\
& Chi-cuadrado aproximado & 9090.458 \\
Prueba de esfericidad de Bartlett & Gl & 861 \\
& Sig. & 0.000 \\
\hline
\end{tabular}

Fuente: Elaboración propia

Ésta Tabla nos muestra dos pruebas de bondad de ajuste, la cual nos muestra que el primero de $\mathrm{KMO}$ es lo ideal, ya que, contiene el valor muy cercano a la unidad (o al $100 \%)$ y que se encuentra representado el 0.974 (97.4\%) de la adecuación de la muestra. Mientras con el segundo, se rechaza la hipótesis nula o se acepta la alternante, por contener, el P-value menor al 5\%, indicándonos que la matriz de correlaciones no es la identidad, es decir, existe la correlación estadísticamente significativa al menos de algunas de ellas (entre los diversos Ítem). Para verificar esa correlación estadísticamente significativa, se plantea la siguiente hipótesis y se contrasta con la prueba estadística de esfericidad de Bartlett.

\section{Discusión}

En la investigación realizada por Descola en el 2000 sobre la relación entre antropología y naturaleza el compromiso de esta ciencia es ayudar a salvar el planeta de su destrucción por todos los grupos sociales; concluye que existe una cultura ambiental ancestral que no es tomada en consideración por los científicos de la oficialidad que esta desencadenando un choque cultural ante la naturaleza.

En las investigaciones realizadas por Miro Quesada en el 2015 sobre ecología práctica sobre la influencia de nuestras costumbres, hábitos, formas de ser y de pensar ante el medio ambiente, son estas lo que no están ayudando a cuidar nuestro habitad; concluyen que debemos cambiar costumbres, hábitos, formas de ser y formas de pensar ante el medio 
ambiente.

De las investigaciones anteriores realizadas por Descola en el 2000; así también por Miro Quesada en el 2015, podemos afirmar que los comportamientos ecológicos tienen influencia significativas positivas y negativas en la cultura ambiental.

En la investigación realizada por Westreicher en el 2016 en "Crisis Ambiental" ello se agudiza por la pobreza que sufre una gran parte de la población en el planeta; concluye que más de tres mil millones de personas viven con menos de dos dólares al día y son más de cuarenta mil niños que cada día mueren por enfermedades relacionadas a la desnutrición. A más pobreza más degradación de la naturaleza. Por lo tanto, se puede inferir de este dato que las condiciones económicas influyen en la manera en que las poblaciones del ámbito rural se vinculan con el medio ambiente.

De las investigaciones de Al Gore en el 2013; así también por Narváez en el 2015, podemos afirmar que los pobladores del distrito de Huancas poseen a través del tiempo un tipo de cultura ambiental que choca con la propuesta oficial de las autoridades que promueven un tipo de defensa del medio ambiente extraño para los habitantes del distrito de Huancas.

\section{CONCLUSIÓN O CONSIDERACIONES FINALES}

- El comportamiento ecológico de los pobladores tiene influencia sobre la cultura ambiental de los pobladores del distrito de Huancas el año 2016.

- Los niveles de motivación del cuidado del medio ambiente de los pobladores del distrito de Huancas son bajas.

- El aspecto económico y los bajos índices de acceso a los servicios educativos influyen negativamente en el comportamiento ambiental de la población. A ello contribuye también la escasa diversificación curricular que se desarrolla en las escuelas. Por lo cual, la pertinencia de la educación se diluye en el contexto.

- La inteligencia ecológica de los pobladores del distrito de Huancas tiene efecto sobre el medio ambiente del distrito de Huancas el año 2016.

\section{LISTA DE REFERENCIAS}

Al, G. (2015). Una verdad incómoda. La crisis planetaria del calentamiento global y cómo afrontarla. España: Gedisa editorial.

Bernales, M. (2006) Artesanos de socavón. Pequeña minería artesanal en América Latina. Perú: Editorial del Congreso del Perú. 
Bonilla, H. (1967) El minero de los andes. Perú: Editorial Instituto de Estudio Peruano.

Burga, M. (1988) Nacimiento de una utopía: muerte y resurrección de los Incas. Perú: Editorial Instituto Apoyo Agrario

Crisanto, F., \& Pinzon, A. A. (2019). Antropología. 21. Universidad Nacional Toribio Rodríguez de Mendoza, Chachapoyas, Perú.

Campbell, B. (1985). Ecología Humana. España: Salvat.

Casado, L. (2010) Resolución de conflictos. Perú: Editorial Punto y Coma.

Dourojeanni, M. J. (2011). Amazonía probable y deseable. Ensayo sobre el presente y futuro de la Amazonía. Universidad Inca Garcilaso de la Vega, Lima, Perú.

Descola, P. (2000). Antropología de la naturaleza. España: Salvat.

Goleman, D. (2009). Inteligencia Ecológica. España: Kairos.

Galindo, Á. (2007). Responsabilidad social corporativa y medios de comunicación social. España: Universidad Pontificia de Salamanca.

Narváez, L. (2015). Los Huancas de Amazonas. Perú: Municipalidad de Huancas. Proyecto sierra Norte. Ministerio de Agricultura, Amazonas, Perú.

Miro Quesada, F. (2015). Ecología práctica. Perú: Grupo El Comercio.

Reátegui, R. (2017). Conga no va. Otro modelo de desarrollo sí es posible. Perú: San Remo.

Spencer, L. N. (2017). Estilo motivacional del docente, tipos de motivación, autoeficacia, compromiso agente y rendimiento en matemáticas en universitarios. Tesis de maestría, Pontificia Universidad Católica del Perú, Lima, Perú.

Sachs, J. (2009). Economía para un planeta abarrotado. Argentina: Debate.

Sachs, J. (2005). El fin de la pobreza. Colombia: Debate.

Vigo, A. (2008) La nueva ruralidad. La campiña de Moche. Perú: Universidad Nacional de Trujillo. 